by day and nystatin ointment at night. When there was local tenderness and increased redness a course of erythromycin was also given, because Staph. pyogenes was always present under these circumstances.

Many treatments have been used in the past and their multiplicity shows that they are often not successful. If this new regimen proves effective it will be a useful advance. Surgical intervention is usually contraindicated, and griseofulvin is no help, as Candida species are not sensitive to this antibiotic.

\section{Lactic Acidosis}

The syndrome of lactic acidosis was first described by W. E. Huckabee, ${ }^{1} 2$ who classified his cases into two groups. In the first the accumulation of lactic acid seemed to be due to accelerated anaerobic glycolysis secondary to hypoxaemia or peripheral circulatory failure. Correction of the defect caused a fall of blood lactate, and the prognosis was good. In the other group, however, there was no obvious cause for the raised lactate concentration, levels were higher (14-26 $\mathrm{m}$-mole/1.), and the outcome was invariably fatal. In such cases there is usually a serious associated condition such as uraemia, ${ }^{3}$ bacterial infection, ${ }^{4}$ alcoholism, ${ }^{5}$ arteriosclerotic heart disease,${ }^{3}$ liver disease,${ }^{6}$ leukaemia, ${ }^{7}$ severe anaemia, ${ }^{8}$ glycogen storage disease, ${ }^{9}$ starvation, ${ }^{10}$ diabetes mellitus, ${ }^{1}$ or phenformin therapy. ${ }^{11}$ Lactic acidosis has also been described as a familial disorder in children with certain neurological disorders $^{12}$ and recently as a chronic disorder in an adult with no evidence of associated disease. ${ }^{13}$

Lactic acid is a terminal product of glycolysis. Since the $\mathrm{pK}$ of lactic acid, $3 \cdot 86$, is well below the $\mathrm{pH}$ of body fluids virtually all lactic acid produced becomes ionized. The lactic acid formed is exclusively the laevo form, but certain tissues, including liver and kidney, can also metabolize the dextro isomer. ${ }^{14}$ Lactate is a "dead end" metabolite and can be metabolized only by reconversion to pyruvate, a reaction catalysed by lactate dehydrogenase and involving coenzyme $\mathrm{I}\left(\mathrm{NAD}^{+}\right)$. Most cell membranes are freely permeable to both lactate and pyruvate, and the blood levels of these metabolites are usually less than 1.5 and $0.2 \mathrm{~m}$-mole/1. respectively. Their half-lives in the circulation are of the order of 10-15 minutes. ${ }^{1516}$

Lactic acidosis may be defined, then, as a raised blood lactate level together with a reduced arterial $\mathrm{pH}$. (This definition excludes certain other causes of raised blood lactate levels including hyperventilation, ${ }^{17}$ obesity, ${ }^{18}$ and the administration of glucocorticoids or oral contraceptives. ${ }^{19}$ ) The incidence of lactic acidosis in a general hospital population is unknown, but it is probably higher than has been suspected. For determination of lactate or pyruvate blood samples must be deproteinized immediately, since the lactate level rapidly rises and the pyruvate falls in vitro. ${ }^{20}$ Specific enzymatic methods ${ }^{21}$ have replaced the older, relatively nonspecific colorimetric techniques. If the lactate level cannot be measured directly lactic acidosis should be suggested by the $^{- \text {the }}$ finding of a large "anion gap" (plasma $\mathrm{Na}^{+}+\mathrm{K}^{+}-\mathrm{Cl}^{-}-$ $\mathrm{HCO}_{3}^{-}$), provided that other causes. such as ketoacidosis, renal failure, and salicylate or methanol intoxication can be eliminated.

It is by no means clear why lactic acidosis should sometimes develop in the clinical disorders listed earlier-a raised blood lactate level is not a usual feature of these conditions. Though by definition patients with idiopathic lactic acidosis have no clinical evidence of impaired tissue perfusion, certain organs could possibly be underperfused even though the blood pressure was normal. Huckabee ${ }^{2}$ produced lactic acidosis experimentally in dogs by induction of pericardial tamponade with no measurable change of cardiac output or blood pressure. It will be important to determine whether the accumulation of lactate in idiopathic lactic acidosis results from over production or underutilization. The metabolic effects extend beyond those of the severe metabolic acidosis, since the ratio of lactate to pyruvate is markedly raised. This suggests a rise in the proportion of reduced to oxidized NAD intracellularly, and the equilibria of other NAD-linked dehydrogenase systems may be disturbed. The concentrations of malate, oxaloacetate, glycerol-3-phosphate, dihydroxyacetone phosphate, 3 phosphoglyceraldehyde, and 1,3-diphosphoglyceric acid may be altered in certain tissues. The effects of these changes on energy metabolism cannot be predicted. P. J. Randle and colleagues ${ }^{22}$ found the pyruvate dehydrogenase system to be inhibited by a rise in proportion of reduced NAD. Since this system represents one of the major routes of lactate disposal a vicious circle of progressive accumulation of lactate may arise.

The treatment of idiopathic lactic acidosis is unsatisfactory, and in view of the high mortality $\left(80-100^{\circ} /{ }^{23}\right)$ several therapeutic approaches have been tried. The mainstays of treatment are correction of any cellular hypoxia when present and administration of bicarbonate. The acidosis has often been surprisingly difficult to correct, and large amounts of bicarbonate may be required. Methylene blue, a redox dye which can serve as an electron acceptor, has been used with some success to correct the abnormal lactate-pyruvate ratio. ${ }^{3}$ Vasoconstrictor drugs should be avoided as they may impair tissue perfusion; indeed there may be a case for the use of $\alpha$ blocking agents to improve tissue perfusion, though such therapy would require careful monitoring of central venous pressure and the administration of blood ro'ume expanders. A combined glucose and insulin regimen led to a rapid fall of blood lactate levels in a single case reported by $\mathbf{H}$. K. Johnson and C. Waterhouse. ${ }^{11}$ It seems clear that correction of the acidosis alone does not prevent a fatal outcome-but the same could be said of diabetic ketosis before the therapeutic use of insulin. Further studies of the aetiology of lactic acidosis are needed so that a rational therapeutic approach may be evolved.

Huckabee, W. E. American Fournal of Medicine, 1961, 30, 833.

2 Huckabee, W. E., American fournal of Medicine, 1961, 30, 840.

Tranquada, R. E., Grant, W. J., and Peterson, C. R., Archives of Internal Medicine, 1966, 117, 192.

+ Waters, W. C., Hall, J. D., and Schwartz, W. B., American fournal of Medicine, $1963,35,781$.

Daughaday, W. H., Lipicky, R. J., and Rasinski, D. C., Nerw England fournal of Medicine, 1962, 267, 1010 .

Bondy, P. K., American Fournal of Medicine, 1963, 24, 428.

C Roth, G. J., and Porte, D., Archives of Internal Medicine, 1970, 125, 317. 77.
Oei, T. L., Clinica Chimica Acta, 1962, 7, 193.

9 Oei, T. L., Clinica Chimica Acta, 1962, 7, 193. Cubberley, P. T., Polster, S. A., and
fournal of Medicine, 1965, 272, 628 .

1 Johnson, H. K., and Waterhouse, C., Archives of Internal Medicine, 1968, 122, 367 .

12 Haworth, J. C., Ford, J. D., and Younoszai, M. K., Canadian Medical Association fournal, 1967, 97, 773.

13 Sussman, K. E., et al., American Fournal of Medicine, 1970, 48, 104.

14 Brin, M., Annals of the New York Academy of Sciences, 1965, 119, 942.

15 Doar, J. W. H., and Cramp, D. G., Clinical Science, 1970, 39, 271.

is Moorhouse, J. A., Lancet, 1964, 1, 689.

17 Hoorhouse, J. A., Lancet, 1964, 1, 689.

17 Huckabee, W. E., Fournal of Clinical Investigation, 1958, 37, $244.17,690$.

18 Doar, J. W. H., Wynn, V., and Cramp, D. G., Metabolism, 1968, 17, 690.

20 Huckabee, W. E., Fournal of Applied Physiology, 1956, 9, 163.

21 Cramp, D. G., Fournal of Clinical Pathology, 1968, 21, 171.

22 Randle, P. J., et al., Recent Progress in Hormone Research, 1966, $22,1$.

is Oliva, P. B., and Schwartz, H. A., Annals of Internal Medicine, 1969, 71, 587. 